\title{
The Effect of Magnet Magnetization Vector Orientation in Halbach Arrays on the Effectiveness of Separation
}

\author{
J. Repková*, M. Mahuliaková, D. Ciprian, M. Lesñák, J. LuñáČeK \\ Department of Physics, VŠB - Technical University of Ostrava, 17. listopadu 15, 70833 Ostrava - Poruba, Czech \\ Republic
}

\begin{abstract}
Magnetic separation is one of the methods that are used to separate magnetic and non-magnetic components from an input mixture. Its effectiveness strongly depends on the strength and distribution of magnetic field in the separator. It is economical to use Halbach permanent magnet arrays to create a suitable magnetic field, since they do not change their properties during separation and do not need electrical energy. During real magnetization process, however, there can be a problem with correct orientation of their magnetization vector. The work based on 2D computer models shows the influence of different orientations of magnetization vectors of individual elements of Halbach arrays on the size and distribution of the magnetic field in the considered separator space. Several model systems that contained four to six typical AlNiCo, SmCo and NdFeB cube shaped magnets with the dimensions $50 \times 50 \times 50 \mathrm{~mm}^{3}$ were used for the analysis.
\end{abstract}

DOI: 10.12693/APhysPolA.126.158

PACS: 07.05.Tp, 07.55.Db, 75.50.Ww

\section{Introduction}

Magnetic separation is an important part of many industrial processes [1]. Sufficiently high values of magnetic fields strengths can be achieved by using permanent magnets. The intensity of magnetic field, the shape and the distribution of magnetic poles influence effectiveness of the separation.

Such magnetic field can be increased by a special arrangement of permanent magnets, as was first proposed by Klaus Halbach in 1985, hence the name of Halbach array [2]. Use of the Halbach arrays in magnetic separators may not only lead to more efficient separation, but also to more effective use of permanent magnets.

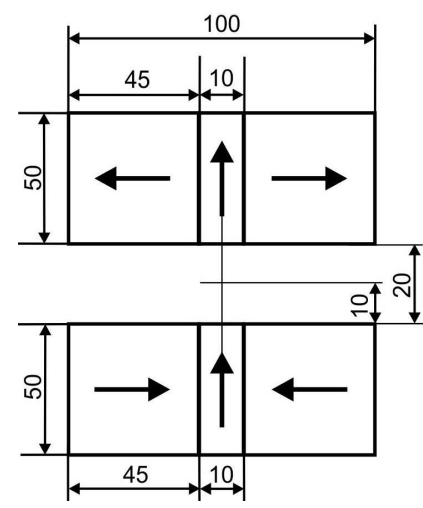

Fig. 1. The classic Halbach three magnet array [1].

This article presents a proposal of geometry optimization of so called trapezoidal configuration in the Halbach

*corresponding author; e-mail: jana.repkova@vsb.cz three-magnet array and the analysis the influence of magnetization vector deviation from an ideal direction on the magnetic field magnitudes (see Fig. 1).

\section{Optimization of Halbach array geometry}

The optimization design uses the classical Halbach array that is shown, including its dimensions, in Fig. 1. It has been known that a stronger magnetic field can be achieved by the change of rectangular magnet shape to so called trapezoidal one [3]. The classic array was substituted by the trapezoidal one and the size of individual elements was optimized, while the inside dimensions and the total volume of magnetic material were kept constant. The resulting array was also augmented by soft magnetic steel segments in order to minimize the stray magnetic flux (see Figs. 2 and 3).

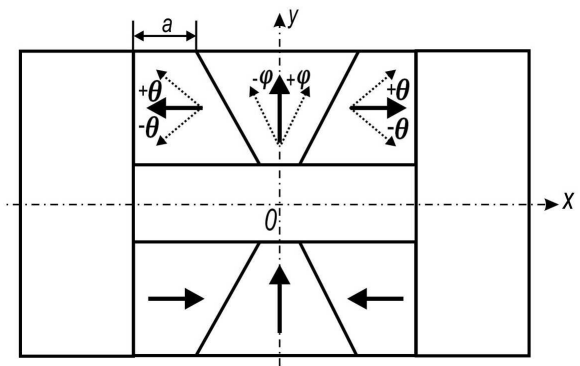

Fig. 2. The optimization of Halbach array with three trapezoidal magnets.

It is rather difficult to get ideal magnetization directions during actual magnetization, as is the case in Figs. 1 and 2. Therefore the influence of magnetization vector misalignment on the value of magnetic field in the middle of air gap was also studied. 


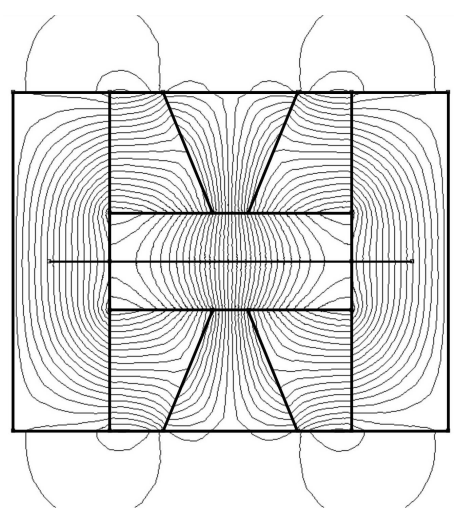

Fig. 3. Distribution of magnetic induction lines in the optimized array.

\section{Results}

The Femm 4.2 software, that can be used with a standard table $\mathrm{PC}$ with a two-core processor and a 32 bit operating system, was used for the modelling of magnetic arrays in $2 \mathrm{D}$.

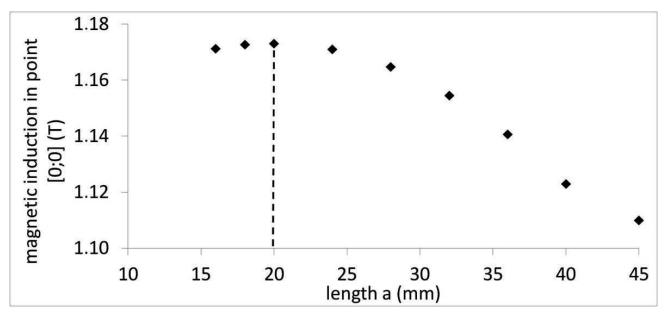

Fig. 4. The influence of central trapezoid outer size on the size of magnetic field.

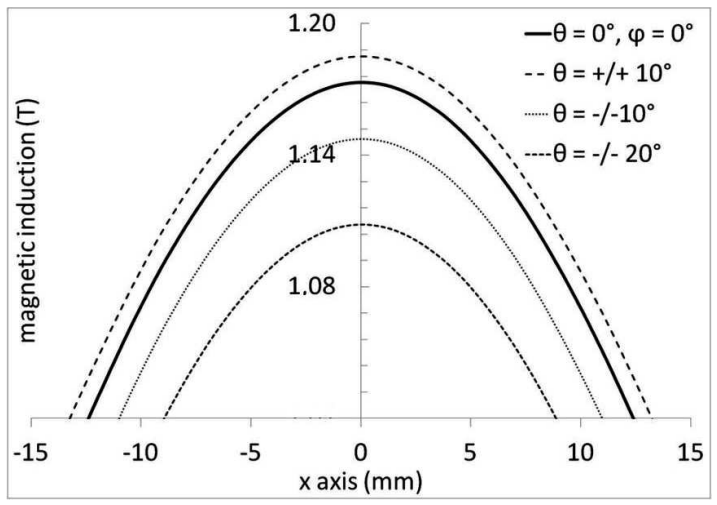

Fig. 5. The influence of magnetization vector orientation on the magnetic field strength - the symmetrical case.

The optimization results for the NdFeB magnet are shown in Figs. 4, 5 and 6. Figure 4 demonstrates the influence of the central trapezoid outer dimension on the size of magnetic field (see Fig. 2). The maximum induction value of $1.173 \mathrm{~T}$ was achieved with the size $a_{\text {opt }}=20 \mathrm{~mm}$. The increase against a standard array with the value of $1.113 \mathrm{~T}$ is $0.060 \mathrm{~T}$.
The influence of magnetization vector orientation was studied for the deviations $\theta= \pm 10^{\circ}$ and $20^{\circ}$ from the horizontal direction of outer segments and $\varphi= \pm 20^{\circ}$ for the middle segment in the upper part of the array (see Fig. 2). The influence of symmetrical changes is seen in Fig. 5 and asymmetrical ones in Fig. 6. The symmetrical change of magnetization (Fig. 5) for the angle of $10^{\circ}$ and $20^{\circ}$ up or down, respectively, leads to the magnetic fields of $1.185 \mathrm{~T}$ and $1.178 \mathrm{~T}$ or $1.147 \mathrm{~T}$ and $1.110 \mathrm{~T}$, respectively. It is clearly seen, the positive vector rotation ("up" direction - see Fig. 2) contributes slightly to the further increase of magnetic induction, whereas the negative rotation in "down" direction leads to its decrease. Figure 6 shows the influence of non-symmetrical changes in magnetization vector orientations. Using these alternatives (non-symmetrical angle changes by $10^{\circ}$ or $20^{\circ}$, respectively), the magnetic fields of $1.167 \mathrm{~T}, 1.150 \mathrm{~T}$ and $1.164 \mathrm{~T}$, in the middle segment of the upper array part, were achieved.

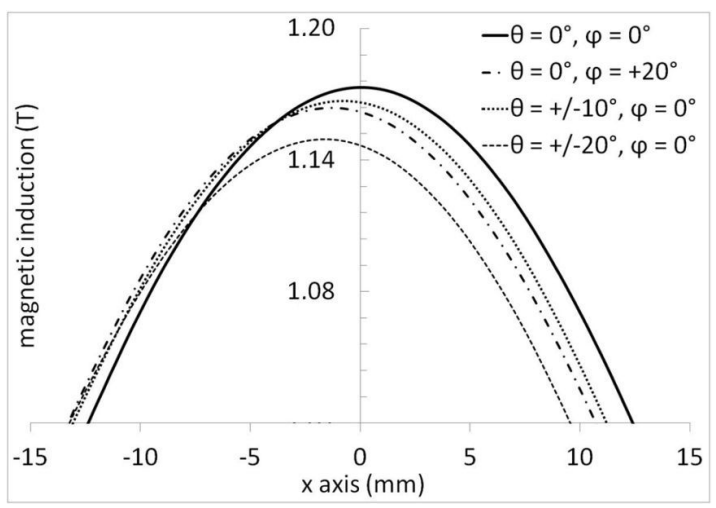

Fig. 6. The influence of magnetization vector orientation on the magnetic field strength - the nonsymmetrical case.

\section{Conclusion}

This work presents the results of dimension optimization of the central segment of Halbach three trapezoidal $\mathrm{NdFeB}$ permanent magnet array and the influence of deviation from an ideal magnetic vector direction. The modeling results will be used to propose a laboratory magnetic separator for separation of sub-microscopic paramagnetic particles.

\section{Acknowledgments}

This article has been supported by the projects SP 2013/7, SP 2103/8 and CZ.1.05/2.1.00/01.0040.

\section{References}

[1] R.K. Dwari, D.S. Rao, P.S.R. Reddy, Int. J. Min. Sci. Technol. 23, 1 (2013).

[2] K. Halbach, J. Appl. Phys. 57, 3605 (1985).

[3] A.E. Marble, IEEE Transactions on Magnetics. 44, 576 (2008). 